MacKay, W.G., Loon, A.M. van, Niedrig, M., Meijer, A., Lina, B., Niesters, H.G.M. Molecular detection and typing of influenza viruses. Are we ready for an influenza pandemic? Journal of Clinical Epidemiology: 2008, 42(2), 194-197

\begin{tabular}{|l|l|}
\hline Postprint Version & 1.0 \\
\hline Journal website & $\underline{\text { http://www.journalofclinicalvirology.com/article/S1386-6532(08)00064- }}$ \\
\hline Pubmed link & $\underline{\underline{\text { http:///www.nct }}}$ \\
\hline DOI & $10.1016 / \mathrm{j} . \mathrm{jcv} .2008 .02 .005$ \\
\hline
\end{tabular}

This is a NIVEL certified Post Print, more info at http://www.nivel.eu

\title{
Molecular detection and typing of influenza viruses: Are we ready for an influenza pandemic?
}

\author{
W.G. MACKAY ${ }^{A,}$, A.M. VAN LOON ${ }^{\mathrm{B}}$, M. NIEDRIG ${ }^{\mathrm{C}}$, A. MEIJER ${ }^{\mathrm{D}}$, B. LINA ${ }^{\mathrm{E}}$ AND H.G.M. NIESTERS ${ }^{\mathrm{F}}$ \\ ${ }^{a}$ The Neutral Office, Quality Control for Molecular Diagnostics (QCMD), Block 4, Kelvin Campus, West of \\ Scotland Science Park, Glasgow G20 0SP, Scotland, UK \\ ${ }^{\mathrm{b}}$ Department of Virology, Eijkman-Winkler Centre, Medical Centre Utrecht, Heidelberglaan 100, 3584 CX \\ Utrecht, The Netherlands \\ ${ }^{c}$ Robert Koch Institute, Nordufer 20, 13353 Berlin, Germany \\ ${ }^{\mathrm{d}}$ European Influenza Surveillance Scheme Co-ordination Centre, Netherlands Institute for Health Services \\ Research (NIVEL), P.O. Box 1568, 3500 BN, Utrecht, The Netherlands \\ ${ }^{\mathrm{e}}$ National Reference Centre for influenza (région sud), Hospices Civils de Lyon and UCBL-CNRS FRE \\ 3011, Université de Lyon, Faculté de médecine R.T.H. Laennec, 69372 Lyon Cedex 08, France \\ fDepartment of Virology, University Medical Centre Groningen, P.O. Box 30.001, 9700 RB, Groningen, The \\ Netherlands
}

\begin{abstract}
Background: We cannot predict when an influenza pandemic will occur or which variant of the virus will cause it. Little information is currently available on the ability of laboratories to detect and subtype influenza viruses including the avian influenza viruses.

Objectives: To assess the ability of laboratories to detect and subtype influenza viruses.

Study design: In 2006 QCMD distributed an External Quality Assessment panel for the molecular detection and haemagglutinin subtyping of influenza viruses to 87 laboratories in 34 countries Worldwide, which were given 6 weeks to return results. These data were analysed to assess laboratory performance.

Results: Influenza virus positive panel samples were correctly identified by $35-98 \%$ of laboratories. The correct haemagglutinin subtype was reported by $32-87 \%$ of laboratories that detected the virus: incorrect subtyping results included the reporting of avian influenza viruses as human strains and vice versa. Twelve laboratories reported false positives with some avian influenza viruses reported.

Conclusions: These data suggest that improvements are needed in the molecular detection of influenza viruses and influenza virus A haemagglutinin subtyping. Only rapid and accurate identification of circulating pandemic influenza virus will ensure that the maximum time is available for intervention.
\end{abstract}

\section{INTRODUCTION}

An influenza pandemic is inevitable but we cannot predict when it will occur or which variant of the virus will be the cause (Rezza, 2004,Webby and Webster, 2003). 
MacKay, W.G., Loon, A.M. van, Niedrig, M., Meijer, A., Lina, B., Niesters, H.G.M. Molecular detection and typing of influenza viruses. Are we ready for an influenza pandemic? Journal of Clinical Epidemiology: 2008, 42(2), 194-197

Influenza virus A (H5N1) is currently of most concern. This avian influenza virus was first reported to infect humans in Hong Kong in 1997 where there were 18 confirmed cases and a mortality rate of 33\% (Claas et al., 1998). To-date influenza virus A (H5N1) has been the causal agent in 317 human cases Worldwide with a mortality rate of $60 \%$ (http://www.who.int/csr/disease/avian_influenza/country/cases_table_2007_06_29/en/index.html). Little information is available on the proficiency of laboratories Worldwide to accurately detect and subtype influenza viruses including the avian influenza viruses, which are considered as a possible cause of future pandemics in humans. One of the best ways of assessing this ability is through participation in external quality assessment (EQA) programmes ([Meijer et al., 2005], [Templeton et al., 2006] and [Valette and Aymard, 2002]). An EQA study was organised by Quality Control for Molecular Diagnostics (QCMD) (http://www.qcmd.org) in collaboration with the European Influenza Surveillance Scheme (EISS) and the European Society for Clinical Virology (ESCV). The aim of this EQA study was to assess the ability of laboratories Worldwide to detect and haemagglutinin subtype influenza viruses using molecular technologies.

\section{METHODS}

The QCMD Influenza virus Haemagglutinin Typing Proficiency Programme panel consisted of 12 samples containing inactivated influenza viruses of the A and B type (of which the A type viruses comprised haemagglutinin subtypes of human and avian origin) and two negative samples. All panel samples were in $0.5 \mathrm{ml}$ of Dulbecco's modified Eagle's medium/10\% Foetal Calf Serum except for influenza virus A (H1N2) and B, which were in $0.5 \mathrm{ml}$ of Viral Transport Medium (Table 1). The panel materials were inactivated by heat and/or irradiation. The materials were analysed by four independent laboratories, recognised as proficient in influenza virus testing using molecular methods, prior to distribution to participating laboratories.

\section{[TABLE 1]}

Laboratories had been invited to participate during early 2006. The randomised EQA panel was distributed on dry ice by QCMD in May 2006 (along with instructions on how the panel samples were to be processed) to 87 laboratories in 34 countries Worldwide (Europe, $n=78$; Asia, $n=4$; Africa, $n=3$; Oceania, $n=2$ ). Laboratories were given 6 weeks to test the panel samples using their routine molecular diagnostic tests for influenza virus and to return their results to QCMD via an online data collection system. They were asked to return qualitative data (presence/absence of influenza virus) and the full haemagglutinin subtype in alpha-numeric format. Laboratories were asked to submit a single dataset for each assay performed. Where laboratories returned multiple datasets (reporting on multiple assays) these data were combined resulting in a single set of results for each laboratory. These results were analysed in order to assess the performance of laboratories in the correct detection, typing and haemagglutinin subtyping of influenza viruses.

\section{RESULTS}

From the 87 laboratories registered for the EQA study, 60 (69\%) laboratories returned a total of 114 datasets (average of two datasets each). Sixteen datasets were removed from the analysis because they reported all panel samples as negative or 'not determined' (meaning that neither a positive or negative result was reported), leaving the total number of datasets analysed at 98 .

Seven (11.6\%) laboratories used commercial assays, 43 (71.7\%) used in-house assays and 10 (16.7\%) used a mixture of both.

Influenza virus positive panel samples were correctly identified by 35-98.3\% of laboratories $($ mean $=70.1 \%)$. The correct type and haemagglutinin subtype was reported by $78-95.7 \%($ mean $=87.6 \%)$ and $31.7-87 \%$ (mean $=55 \%$ ) of laboratories that detected the virus. The percentage of laboratories correctly detecting influenza virus was directly proportional to the viral load of the panel samples, with the percentage of laboratories that correctly subtyped the influenza A-type viruses being much less (Table 1).

Two avian influenza viruses were represented in the EQA panel (A (H5) and A (H7)). The influenza virus A (H5) panel sample of highest viral load (approximately 9600 copies/ml) was correctly subtyped by $87.0 \%$ 
MacKay, W.G., Loon, A.M. van, Niedrig, M., Meijer, A., Lina, B., Niesters, H.G.M. Molecular detection and typing of influenza viruses. Are we ready for an influenza pandemic? Journal of Clinical Epidemiology: 2008, 42(2), 194-197

( $n=40 / 46)$ of laboratories that detected influenza virus. One laboratory incorrectly reported that the panel sample contained influenza virus A (H1). Five laboratories detected the virus but reported no subtype. The influenza virus A (H7) panel sample (approximately 4500 copies/ml) was correctly subtyped by $31.7 \%$ ( $n=13 / 41$ ) of laboratories that detected the virus: five laboratories incorrectly reported that the panel sample contained other influenza viruses (A H3 (n= 2), A H5 (n=1), A H3 and B (n=1), B (n=1)). Twenty-three laboratories detected an influenza virus in the panel sample but reported no subtype.

Two currently circulating human influenza virus A subtypes were represented in the EQA panel (A H1 and A H3). The influenza virus A (H1) panel sample (approximately 188,000 copies/ml) was correctly subtyped by $54.2 \%(n=32 / 59)$ of laboratories that detected the virus. Five laboratories incorrectly reported that the panel sample contained other influenza viruses (A H3 ( $n=1)$, A H5 (n= 1), A H1 and H5 (n= 2), A H1 and B $(n=1)$ ). Twenty-two laboratories detected the virus but reported no subtype. The influenza virus A (H3) panel sample of highest viral load (approximately 20,000 copies/ml) was correctly subtyped by $51.7 \%(n=30 / 58)$ of laboratories that detected the virus: four laboratories incorrectly reported that the panel sample contained other influenza viruses (A H1 ( $n=1)$, A H5 (n=1), A H1 and H5 (n= 1), A H3 and H5 $(n=1)$ ). Twenty-four laboratories detected the virus but reported no subtype.

The two influenza virus negative panel samples were incorrectly reported 15 times. There were 13 (10.8\%) false positive results (reported by 12 laboratories) and two 'not determined' results (reported by two laboratories). Three laboratories reported that these panel samples contained influenza virus A (H5) (Table 1).

\section{DisCUSSION}

Rapid and accurate detection of candidate pandemic influenza virus strains currently circulating in humans is absolutely necessary for the surveillance and control of pandemic influenza (Li et al., 2004). Incorrect diagnosis of a pandemic strain, as a currently circulating influenza virus, would delay intervention and reduce the likelihood of successful containment. The incorrect diagnosis of a candidate pandemic strain, in a case of non-pandemic influenza, would also have serious consequences for the country involved.

This study clearly demonstrated that while laboratories generally showed competence in detecting the presence of influenza virus many did not report the correct haemagglutinin subtype of influenza virus, either of the subtypes currently circulating in humans (influenza virus A H1 or A H3) or of the subtype considered as a risk for a future human pandemic (influenza virus A H5). Incorrect subtyping of influenza viruses was common and included the improper reporting of avian influenza viruses as human strains and vice versa. In addition, this study clearly showed that the ability of laboratories to correctly detect and subtype influenza viruses dropped rapidly with decreasing viral load. These observations may be explained by an incomplete match of the primers and/or probes used for the full range of influenza virus A haemagglutinin subtypes represented in the panel.

False positivity was also high with some avian influenza viruses reported. This may have been the result of contamination occurring in the laboratories concerned. There were no false positive results reported by the independent testing laboratories.

Laboratories must improve their performance in the sensitive detection and correct subtyping of influenza viruses so that inaccuracies in diagnosis can be avoided. This will only be achieved if laboratories have access to the most recent information on circulating strains and proper international reference materials for avian influenza viruses. Laboratories must also monitor their performance regularly and participation in international EQA programmes is an important part of this.

\section{ACKNOWLEDGEMENTS}

QCMD would like to thank all of the laboratories who participated in this study. QCMD also wishes to acknowledge the support of J. Ellis and M. Zambon (Health Protection Agency, London, UK), B. Schweiger (Robert Koch Institute, Berlin, Germany) and O. Hungnes (Norwegian Institute of Public Health, Oslo, Norway) in performing independent testing. QCMD also wishes to thank Dr. Harry Staines (Sigma Statistical Services, Balmullo, Scotland) for statistical support. 
MacKay, W.G., Loon, A.M. van, Niedrig, M., Meijer, A., Lina, B., Niesters, H.G.M. Molecular detection and typing of influenza viruses. Are we ready for an influenza pandemic? Journal of Clinical Epidemiology: 2008, 42(2), 194-197

\section{REFERENCES}

Claas et al., 1998 E.C.J. Claas, A.D.M.E. Osterhaus, R. van Beek, J.C. de Jong, G.F. Rimmelzwaan and D.A. Senne et al., Human influenza A H5N1 virus related to a highly pathogenic avian influenza virus, Lancet 351 (1998), pp. 472-477.

Li et al., 2004 K.S. Li, Y. Guan, J. Wang, G.J.D. Smith, K.M. Xu and L. Duan et al., Peiris JSM. Genesis of a highly pathogenic and potentially pandemic H5N1 influenza virus in eastern Asia, Nature 430 (2004), pp. 209-213.

Meijer et al., 2005 A. Meijer, M. Valette, J.C. Manuguerra, P. Perez-Brena, J. Paget and C. Brown et al., Virology Working Group of the European Influenza Surveillance Scheme: implementation of the community network of reference laboratories for human influenza in Europe, J Clin Virol 34 (2005), pp. 87-96.

Rezza, 2004 G. Rezza, Avian influenza: a human pandemic threat?, J Epidemiol Comm H 58 (2004), pp. 807-808.

Templeton et al., 2006 K.E. Templeton, C.B. Forde, A.M. van Loon, E.C.J. Claas, H.G.M. Niesters and P. Wallace et al., A multi-centre pilot proficiency programme to assess the quality of molecular detection of respiratory viruses, J Clin Virol 35 (2006), pp. 51-58.

Valette and Aymard, 2002 M. Valette and M. Aymard, Quality control assessment of influenza and RSV testing in Europe: 2000-01 season, Eurosurveillance 7 (2002), pp. 161-165.

Webby and Webster, 2003 R.J. Webby and R.G. Webster, Are we ready for pandemic influenza?, Science 302 (2003), pp. 1519-1522.

\section{TABLE}

Table 1

Summary of the results returned by laboratories $(n=60)$

\begin{tabular}{|c|c|c|c|c|c|c|c|c|}
\hline \multirow[t]{2}{*}{ Sample content ${ }^{\mathrm{a}}$} & \multirow[t]{2}{*}{ Stock dilution } & \multirow[t]{2}{*}{$\begin{array}{l}\text { Viral load } \\
\text { copies/ml }\end{array}$} & \multicolumn{2}{|c|}{ Correct detection } & \multicolumn{2}{|c|}{$\begin{array}{l}\text { Correct type (A or B) } \\
\text { reported }^{\mathrm{c}, \mathrm{d}}\end{array}$} & \multicolumn{2}{|c|}{$\begin{array}{l}\text { Correct subtype } \\
\text { reported }^{c}\end{array}$} \\
\hline & & & $n$ & $\%$ & $n$ & $\%$ & $n$ & $\%$ \\
\hline Influenza virus $\mathrm{A}, \mathrm{H} 1$ & $1.0 \times 10^{-2}$ & 188,000 & 59 & 98.3 & 53 & 89.8 & 32 & 54.2 \\
\hline Influenza virus $\mathrm{A}, \mathrm{H} 3$ & $2.0 \times 10^{-5}$ & 20,000 & 58 & 96.7 & 51 & 87.9 & 30 & 51.7 \\
\hline Influenza virus $\mathrm{A}, \mathrm{H} 3$ & $2.0 \times 10^{-6}$ & 2,200 & 46 & 76.7 & 39 & 84.8 & 17 & 37.0 \\
\hline Influenza virus $\mathrm{A}, \mathrm{H} 3$ & $1.0 \times 10^{-6}$ & 1,200 & 39 & 65.0 & 33 & 84.6 & 16 & 41.0 \\
\hline Influenza virus $\mathrm{A}, \mathrm{H} 3$ & $2.0 \times 10^{-7}$ & 200 & 21 & 35.0 & 18 & 85.7 & 7 & 33.3 \\
\hline Influenza virus $\mathrm{A}, \mathrm{H} 5$ & $1.0 \times 10^{-3}$ & 9,600 & 46 & 76.7 & 44 & 95.7 & 40 & 87.0 \\
\hline Influenza virus $\mathrm{A}, \mathrm{H} 5$ & $2.0 \times 10^{-4}$ & 1,600 & 42 & 70.0 & 38 & 90.5 & 31 & 73.8 \\
\hline Influenza virus $\mathrm{A}, \mathrm{H} 5$ & $1.0 \times 10^{-4}$ & 1,340 & 40 & 66.7 & 37 & 92.5 & 27 & 67.5 \\
\hline Influenza virus $\mathrm{A}, \mathrm{H} 5$ & $1.0 \times 10^{-4}$ & 1,460 & 38 & 63.3 & 33 & 86.8 & 25 & 65.8 \\
\hline Influenza virus $\mathrm{A}, \mathrm{H} 5$ & $2.0 \times 10^{-5}$ & 130 & 29 & 48.3 & 26 & 89.7 & 18 & 62.1 \\
\hline Influenza virus $\mathrm{A}, \mathrm{H} 7$ & $4.0 \times 10^{-5}$ & 4,500 & 41 & 68.3 & 32 & 78.0 & 13 & 31.7 \\
\hline Influenza virus B & $1.0 \times 10^{-2}$ & 22,717 & 46 & 76.7 & 39 & 84.8 & & \\
\hline Negative & - & - & 53 & 88.3 & & & & \\
\hline Negative & - & - & 52 & 86.7 & & & & \\
\hline
\end{tabular}

